\title{
OCT-Precoding Combined with LDPC-Coding Scheme for 128 QAM MB-OFDM UWBoF System
}

\author{
Changqing Xiang $\mathbb{D}^{1}{ }^{1}$ Xi Yang $\mathbb{D},{ }^{1}$ Xiaojun $\mathrm{Zhu},{ }^{2}$ Qiong He, ${ }^{1}$ Renmin Zhang, \\ and Zhiyang Xiang ${ }^{1}$ \\ ${ }^{1}$ College of Information Science and Engineering, Jishou University, Jishou, Hunan 416000, China \\ ${ }^{2}$ School of Software Engineering, Jiangxi University of Science and Technology, Nanchang, Jiangxi 330013, China \\ Correspondence should be addressed to Xi Yang; ynkej@163.com
}

Received 3 August 2020; Revised 3 October 2020; Accepted 29 October 2020; Published 29 November 2020

Academic Editor: Junmin Liu

Copyright (c) 2020 Changqing Xiang et al. This is an open access article distributed under the Creative Commons Attribution License, which permits unrestricted use, distribution, and reproduction in any medium, provided the original work is properly cited.

In this paper, we proposed a coding scheme based on orthogonal cyclic transpose (OCT) precoding combined with low-density parity check (LDPC) code to improve the transmission performance of 128 -quadrature amplitude modulation multiband orthogonal-frequency-division-multiplexing ultrawide band over fiber (128 QAM MB-OFDM UWBoF) system. The proposed scheme could not only improve the performance of the system but also average the signal-to-noise ratio (SNR) of subcarriers and reduce the difference among subchannels in practical application. The simulation results showed that compared with the traditional scheme, the receiver performance of this scheme was improved by $2.7 \mathrm{~dB}$ when the LDPC-coding rate was $87.5 \%$ through $70 \mathrm{~km}$ standard single mode fiber (SSMF) transmission and the bit error rate (BER) was $3.8 \times 10^{-3}$. Besides, the constellation distribution was more convergent and uniform.

\section{Introduction}

MB-OFDM UWB signal is widely used in access networks due to its notable advantages such as low power consumption, high bandwidth, and antielectromagnetic interference [1-3]. It is precisely because of the rapid development of broadband communication and network technology that the communication demand has developed from simple voice services to higher-demand broadband services such as data and high-definition video transmission. In order to expand the coverage area of MB-OFDM UWB signals, MB-OFDM UWB signals combined with optical fiber communication systems have attracted extensive attention [4-6].

Recently, the proposal of OCT-precoding scheme has improved the error code performance and equalized the signal-to-noise ratio of each data subcarrier in the signal [7-9]. Then, the scheme based on the combination of OCTprecoding and adaptive modulation OFDM was studied in the fiber fusion laser visible light system [10]. OFDM signals based on rise code (RS) combined with the OCT-precoding scheme have been investigated in the fiber fusion laser visible light system. BER performance could be improved, and SNR on each subcarrier could be averaged by using the scheme based on OCT-precoding combined with adaptive modulation OFDM and the scheme based on RS code combined with OCT-precoding, which has been proved in the fiber fusion laser visible light system [11]. For MB-OFDM UWB signals, in the process of optical fiber transmission, it is easy to be affected by the nonlinearity of optical devices and optical fibers, especially the noise of modulators and amplifiers, chromaticity dispersion, and insertion loss of optical fibers. In order to overcome the signal deterioration caused by the above problems, dispersion precompensation scheme, intrasymbol frequency-domain averaging (ISFA) adaptive selection of the best window scheme, and LDPC-coding forward error correction technology have been applied to MB-OFDM UWB systems [12-14], which are committed to improving the transmission performance in optical fiber systems. 
In this paper, the OCT-precoding scheme is used to average the SNR of subcarriers of 128 QAM MB-OFDM UWB signals, so as to reduce the channel difference among each subchannel. In order to further improve the system performance, the proposed OCT-precoding combined with the LDPC-coding scheme is compared with the traditional scheme. Compared with the traditional scheme, the BER performance is improved by $2.7 \mathrm{~dB}$ under the condition of BER of $3.8 \times 10^{-3}$ through $70 \mathrm{~km}$ SSMF transmission, and the LDPC-coding rate used in this paper is $87.5 \%$.

\section{Principle of OCT-Precoding}

In order to optimize the transmission performance of the MB-OFDM UWBoF system, we consider the OCT-precoding scheme. The specific implementation is to perform an OCT-precoding process on the transmitted data after QAM symbol mapping, where the precoding scheme is to multiply the signal data by a Zadoff-Chu (ZC) sequence. The expression of this sequence matrix is as follows:

$$
F=\frac{1}{\sqrt{N}} \times\left[c_{1}, c_{2}, \ldots, c_{N} ; c_{N}, c_{1}, \ldots, c_{N-1} ; \ldots ; c_{2}, c_{3}, \ldots, c_{1}\right]
$$

where $N=100$ represents the total number of data subcarriers and $\left[c_{1}, c_{2}, \ldots, c_{N}\right]$ represents the ZC sequence, corresponding to each element. The $\mathrm{ZC}$ sequence can be obtained by the following expression:

$$
Y_{u}(m)= \begin{cases}e^{-j\left(\pi(u t)(t+1+2 q) / N_{\mathrm{zc}}\right)}, & N_{\mathrm{zc}} \in \text { odd }, \\ e^{-j\left(\pi(u t)(t+2 q) / N_{\mathrm{zc}}\right)}, & N_{\mathrm{zc}} \in \text { even. }\end{cases}
$$

Here, $0<u<N_{\mathrm{zc}}, \quad 0 \leq t<N_{\mathrm{zc}}, \operatorname{gcd}\left(N_{\mathrm{zc}}, u\right)=1$ is the greatest common divisor, $q$ is an integer, and $N_{z c}$ represents the appropriate length $N$ of the OCT-precoding sequence.

Here, the modulus of $c_{i}$ is 1 . The length $N_{z c}$ of the ZC sequence has good periodic autocorrelation, and its periodic autocorrelation function is

$$
\begin{aligned}
A C(p) & =\sum_{k=0}^{N-1} c_{k} c_{T}^{*} \\
& = \begin{cases}E, & p=0\left(\bmod N_{\mathrm{zc}}\right), \\
0, & p \neq 0\left(\bmod N_{\mathrm{zc}}\right),\end{cases}
\end{aligned}
$$

wherein, (.)*stands for conjugate transpose operation, $E=A C(0)$, expectation is 0 , So $F$ is an orthogonal matrix, and $F^{*}=F^{-1}$. Multiplying MB-OFDM UWB data symbol and $F$, we can get

$$
\begin{aligned}
S_{\text {signal }}=\left(X_{1}, X_{2}, \ldots, X_{N}\right) \times F \\
=\left(\frac{1}{\sqrt{N}}\right)\left[\begin{array}{c}
c_{1} X_{1}+c_{N} X_{2}+\cdots+c_{2} X_{N} \\
c_{2} X_{1}+c_{1} X_{2}+\cdots+c_{3} X_{N} \\
\vdots \\
c_{N} X_{1}+c_{N-1} X_{2}+\cdots+c_{1} X_{N}
\end{array}\right] .
\end{aligned}
$$

After transmission, the received signal before decoding can be expressed as

$$
S_{\text {signal }}+S_{\text {noise }}=\left(\frac{1}{\sqrt{N}}\right)\left[\begin{array}{c}
c_{1} X_{1}+c_{N} X_{2}+\cdots+c_{2} X_{N}, \\
c_{2} X_{1}+c_{1} X_{2}+\cdots+c_{3} X_{N}, \\
\vdots \\
c_{N} X_{1}+c_{N-1} X_{2}+\cdots+c_{1} X_{N}
\end{array}\right]+\left[\begin{array}{c}
t_{1} \\
t_{2} \\
\vdots \\
t_{N}
\end{array}\right] \text {, }
$$

where $t_{i}$ represents the noise on the data subcarrier; then, extract the received data and multiply it by $F^{-1}$. The result can be expressed as

$$
\begin{aligned}
R_{r x} & =\left(S_{\text {signal }}+S_{\text {noise }}\right) \times F^{-1} \\
& =\left[\begin{array}{c}
X_{1} \\
X_{2} \\
\vdots \\
X_{N}
\end{array}\right]+\left(\frac{1}{N}\right)\left[\begin{array}{c}
c_{1}^{*} t_{1}+c_{2}^{*} t_{2}+\cdots+c_{N}^{*} t_{N}, \\
c_{N}^{*} t_{1}+c_{1}^{*} t_{2}+\cdots+c_{N-1}^{*} t_{N}, \\
\vdots \\
c_{N-1}^{*} t_{1}+c_{N}^{*} t_{2}+\cdots+c_{1}^{*} t_{N}
\end{array}\right] .
\end{aligned}
$$

We see that the modulus of each element of the Zadoff-Chu sequence is 1 , and the noise of each carrier is relatively balanced, which leads to the balance of the signalto-noise ratio in the MB-OFDM UWB signal.

In IEEE 802.15.3c [14], a detailed introduction to the structure and algorithm of LDPC codes is given, as well as check matrix parameters that have outstanding performance and are easy to implement under different code rates and different structures. Due to the sparse nature of the parity check matrix, when LDPC-coding is grouped, the number of bits of data information in the same check matrix is scattered and small, making LDPC-coding resistant to burst continuous errors, and it can be efficiently decoding. Therefore, in the MB-OFDM UWBoF communication system, based on the LDPC-coding scheme, the system performance can be improved while not bringing too much change to the computational complexity of the system.

\section{Simulation System Settings}

The simulation setup of a 128 QAM MB-OFDM UWBoF transmission system based on OCT-precoding combined with the LDPC-coding scheme is shown in Figure 1. At the transmitter, in order to avoid the emergence of continuous error bursts, the generated pseudorandom binary sequence (PRBS) passed through the bit interleaver and was scrambled, and we adopted the bit data stream output from the bit interleaver to use a coding rate of 7/8 LDPC-precoding scheme for processing. The encoding method based on LDPC encoding used here was strictly in accordance with the IEEE802.15.3.c standard [14]. Then, the output bit stream was mapped to generate 128-order QAM symbols, and then OCT-precoding was performed, and 12 pilot symbols with virtual subcarriers were inserted and sent. Three TSs were added to the three subbands, respectively 


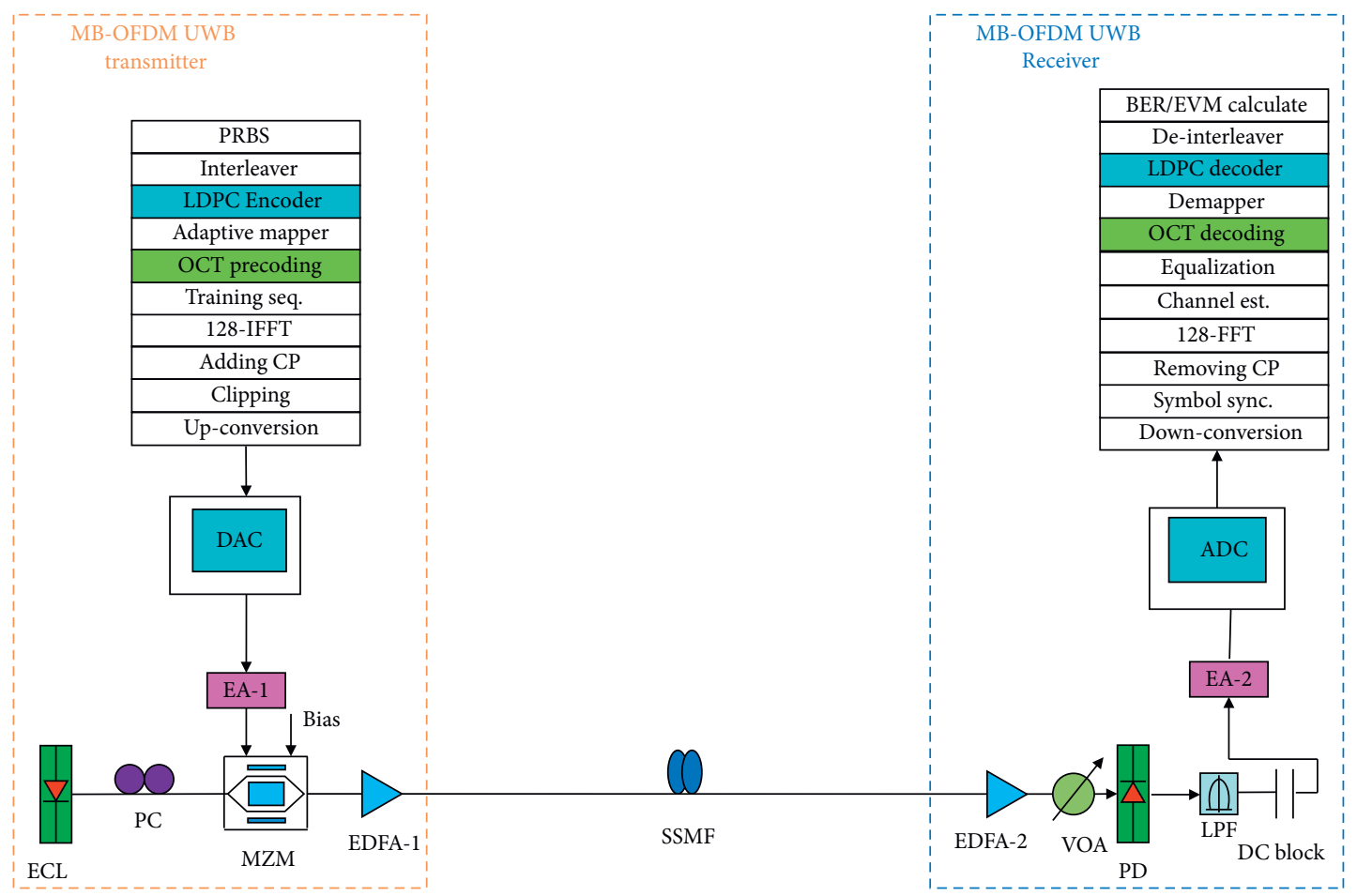

FIGURE 1: System simulation device diagram based on OCT-precoding combined with the LDPC-coding scheme.

[12]. The TSs on the first subbands were used for symbol synchronization and for estimating the channel of the first subband. The latter two were used to estimate the channels of the second and third subbands, respectively. Then, we used 128-point inverse fast Fourier transform (IFFT) to implement subcarrier OFDM modulation. In order to reduce the intersymbol interference in the fiber channel, we added a cyclic prefix of a specific length of 16 . We limited the OFDM signal, and the limiting ratio was $13 \mathrm{~dB}$. And the baseband OFDM signal was digitally upconverted to the MB-OFDM UWB signal through time-frequency coding (TFC). Then, the signal was imported into OptiSystem simulation software from the MATLAB input window, and digital-to-analog conversion was performed for analog signal output for optical fiber transmission.

At the receiver, we exported the captured signal through the MATLAB output window for further offline digital signal processing (DSP). We digitally downconvert the obtained MB-OFDM UWB digital signal to a baseband OFDM signal. Next, symbol synchronization was achieved by using the first TSs, then the CP data at the beginning of the OFDM symbol was removed, and the demodulation of the OFDM signal was completed by a 128-point fast Fourier transform (FFT) operation. The channel responses on the corresponding three subbands were estimated by extracting the three TSs. Subsequently, the channel response on each data subcarrier was equalized, and then the OCT-decoding process was performed and demapped into the original transmission bit stream. The original bit stream after demapping was imported into the LDPC decoder for decoding, and the obtained OFDM output signal was sent to the de-interleaver for de-interleaving processing, and finally the bit error rate was calculated. The key parameters of 128 QAM MB-OFDM UWBoF system simulation based on OCT-precoding combined with the LDPC-coding scheme are listed in Table 1.

\section{Simulation Results and Analysis}

Figure 2 shows the BER performance of the 128 QAM MBOFDM UWB signal based on the traditional scheme and the OCT-precoding scheme after $70 \mathrm{~km}$ SSMF transmission. It could be seen from Figure 2 that the OCT-based precoding scheme did not significantly improve the BER. Compared with the traditional scheme, the BER performance of the OCT-precoding scheme would be improved when the received optical power was higher. When the received optical power was low, the performance would deteriorate. This may be due to the fact that the OCT-precoding scheme is an average subcarrier signal-to-noise ratio (SNR) algorithm, and therefore, it does not essentially promote the BER itself. In other words, the OCT-precoding scheme could make the BER performance better when it is good, and vice versa. It could also be seen from Figure 2 that only when the received optical power was greater than a certain value (between $-9 \mathrm{dBm}$ and $-8 \mathrm{dBm})$, the OCT-precoding scheme could improve the BER performance at this time. Moreover, as the received optical power increased, the OCT-based precoding scheme improved the BER performance more obviously.

Next, we considered the BER performance on the three subbands. The results of the simulated BER performance on the three subbands after $70 \mathrm{~km}$ SSMF transmission, the traditional scheme, and the 128 QAM MB-OFDM UWBoF system based on the OCT-precoding scheme are shown in 
TABLE 1: The main parameters of simulation based on OCT-precoding combined with the LDPC-coding scheme.

\begin{tabular}{lc}
\hline Parameter name & Parameter name \\
\hline OFDM symbol duration & $312.5 \mathrm{~ns}$ \\
Number of IFFT/FFT points/number of subcarriers & 128 \\
CP and ZPS & $37(=16+21)$ \\
Data/pilot/empty subcarrier/protection & $100 / 12 / 6 / 10$ \\
Bandwidth (only for one frequency band) & $528 \mathrm{MHz}$ \\
Number of TS/OFDM symbols & $3 / 312$ \\
DAC and ADC sampling rate & $10.561 \mathrm{GSps}$ and $20 \mathrm{GSps}$ \\
Modulation scheme & $128 \mathrm{QAM}$ \\
Total number of bits per OFDM symbol & $128 \mathrm{QAM}: 100 * 7=700 \mathrm{bits}$ \\
Original signal bit rate & $128 \mathrm{QAM}: \log _{2}(128) *(1 / 312.5) * 100=2.24 \mathrm{Gbps}$ \\
Net signal bit rate & $128 \mathrm{QAM}: 700 * 312 /(315 * 165 * 20 / 10.561)=2.22 \mathrm{Gbps}$ \\
Spectral efficiency & $128 \mathrm{QAM}: 2.22 /(100 / 128 * 0.528)=5.38 \mathrm{bit} / \mathrm{s} / \mathrm{Hz}$ \\
LDPC-coding rate & $87.5 \%$ \\
Net signal bit rate (LDPC coding) & $2.24 * 87.5 \% \mathrm{Gbps}=1.95 \mathrm{Gbps}$ \\
Spectral efficiency (LDPC coding) & $5.38 * 87.5 \% \mathrm{bit} / \mathrm{s} / \mathrm{Hz}=4.71 \mathrm{bit} / \mathrm{s} / \mathrm{Hz}$ \\
\hline
\end{tabular}

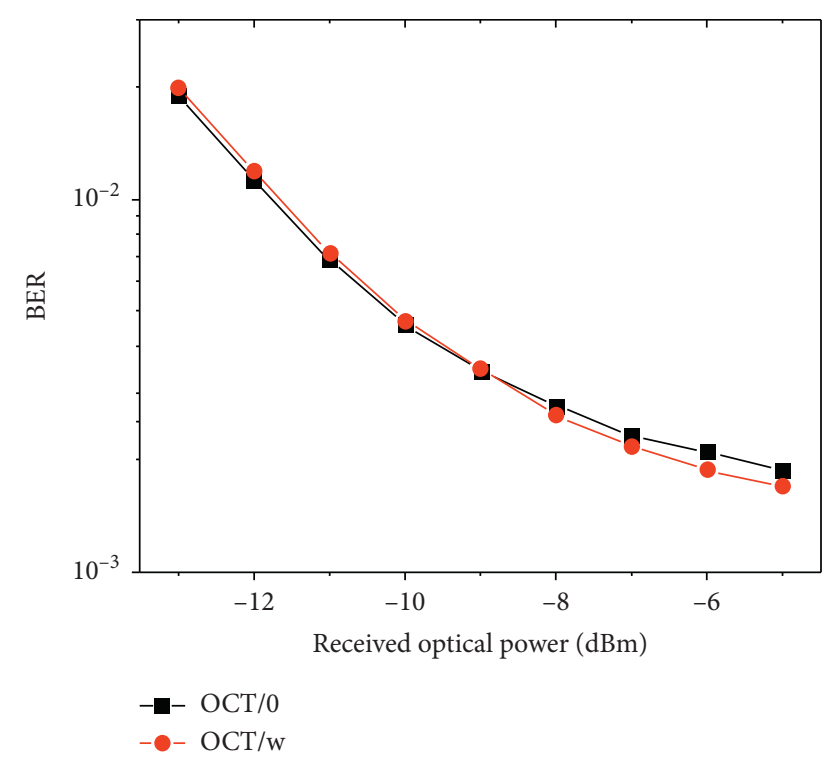

FIGURE 2: BER performance based on the OCT-precoding scheme.

Figure 3. We selected a curve of the OCT-precoding scheme to improve BER performance for discussion. It could be seen from Figure 3 that, under the traditional scheme, the BER performance of the three subbands was the best in the first subband, better in the second subband, and worst in the third subband. After implementing the OCT-precoding scheme, the first subband presented the most obvious improvement in BER, the second subband was worse, and the third subband exhibited the least improvement. Moreover, when the OCT-precoding scheme could improve BER performance, the corresponding received optical power threshold also changed. When the received optical power was greater than about $-12 \mathrm{dBm}$, the OCT-based precoding scheme could improve the BER of the first subband. When the received optical power was greater than about $-10 \mathrm{dBm}$, the OCT-precoding scheme could enable the BER of the second subband to be improved. If the BER of the third subband was to be improved based on the OCT-precoding scheme, the received optical power should be greater than

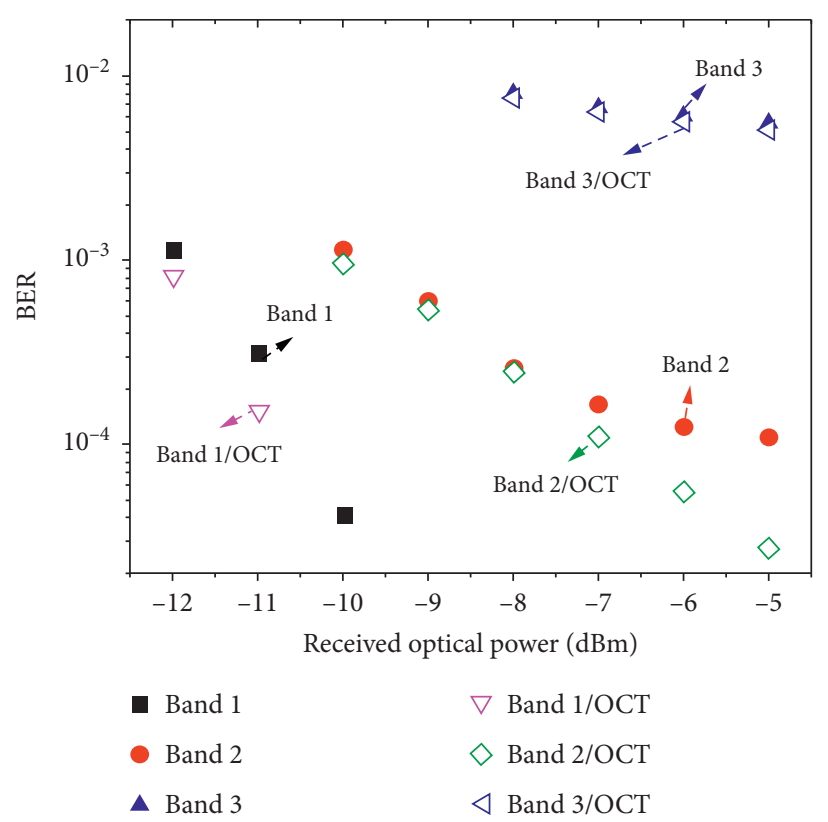

FIGURE 3: BER performance of the three subbands based on the OCT-precoding scheme.

about $-8 \mathrm{dBm}$. And the higher the received optical power of the three sub-bands is, the more the BER improved.

Figure 4 shows the SNR curve of each subcarrier in the traditional scheme and 128 QAM MB-OFDM UWB signal based on the OCT-precoding scheme when the received optical power was $-5 \mathrm{dBm}$ after a simulation of $70 \mathrm{~km} \mathrm{SSMF}$ transmission. Based on the traditional scheme, the SNR curve of each subcarrier is more discrete, with more glitches and sharper peaks. Based on the OCT-precoding scheme, the SNR curve of each subcarrier become relatively concentrated, relatively flat, and the SNR among each subcarrier was not much different. The biggest advantage of the OCTprecoding scheme was that it could average the SNR of the subcarriers. Although it did not greatly improve the BER performance, in practical applications, the OCT-precoding scheme could balance the information given to each user and 


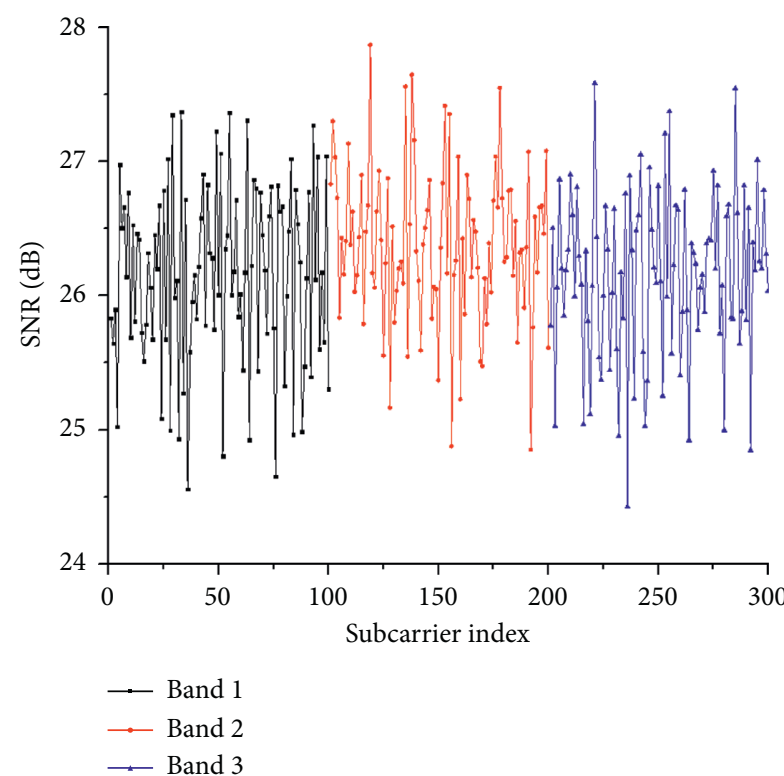

(a)

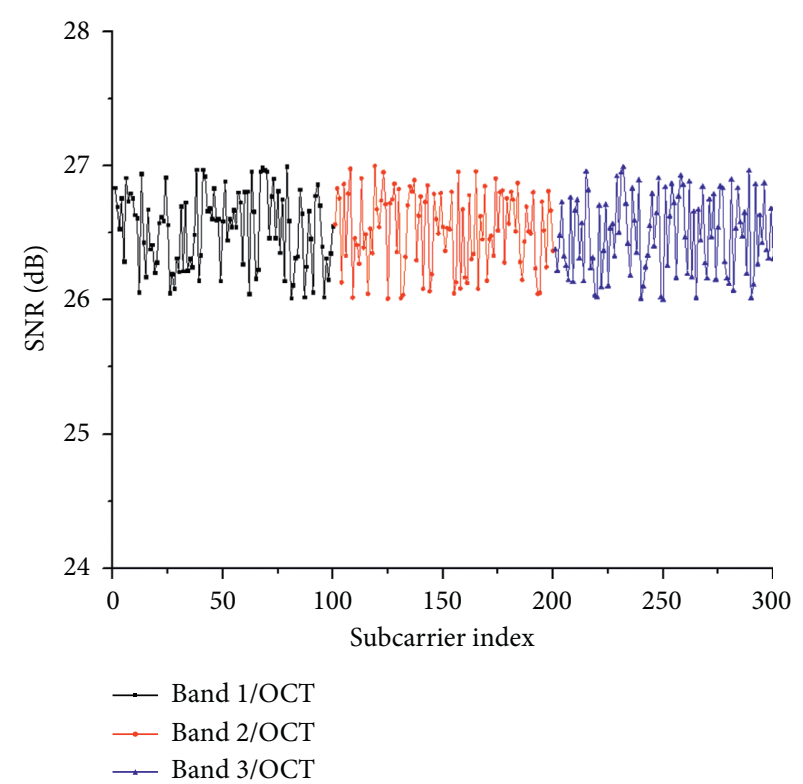

(b)

Figure 4: SNR of each subcarrier of the 128 QAM MB-OFDM UWBoF system.

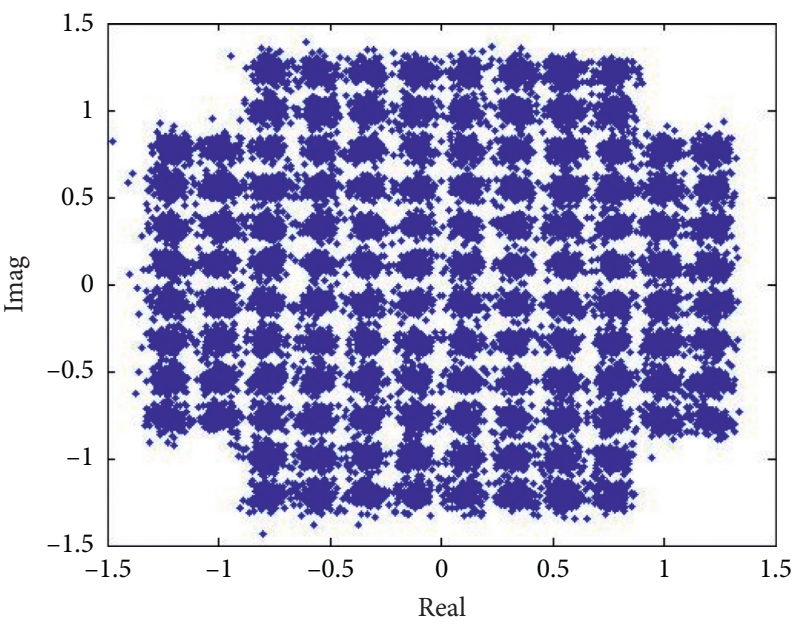

(a)

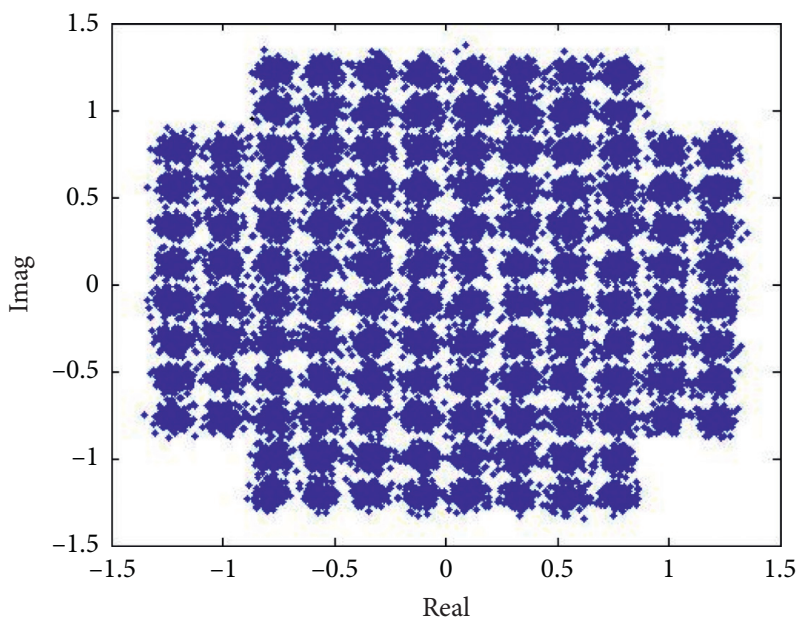

(b)

FIgURE 5: Constellation diagram of the 128 QAM MB-OFDM UWB signal.

reduce the difference among various channels, which is practically important.

The simulation showed the constellation diagram of 128 QAM MB-OFDM UWB signal based on the traditional scheme (left) and the OCT-precoding scheme (right) after $70 \mathrm{~km}$ SSMF transmission, as shown in Figure 5. At this time, the received optical power corresponding to the constellation diagram was $-5 \mathrm{dBm}$. It could be seen from Figure 5 that the constellation diagrams of the 128 QAM MB-OFDM UWB signal based on the traditional scheme and the OCT-precoding scheme were not much different, but the points on the constellation diagram of the latter were more convergent, especially in the peripheral constellation points. Overall, the constellation diagram of 128
QAM MB-OFDM UWB signals based on the OCT-precoding scheme was more evenly distributed.

Figure 6 shows the simulated BER performance of 128 QAM MB-OFDM UWB signals after $70 \mathrm{~km}$ SSMF transmission, traditional scheme, and OCT-based precoding scheme and OCT-based precoding combined with the LDPC-coding scheme. As can be seen from Figure 6, when the BER was $3.8 \times 10^{-3}$, the reception sensitivity of 128 QAM MB-OFDM UWB signals based on OCT-precoding combined with the LDPC-coding scheme was improved by about $2.7 \mathrm{~dB}$ compared with OCT-precoding program. Compared with the traditional scheme, the 128 QAM MB-OFDM UWB signal based on OCT-precoding combined with the LDPCcoding scheme not only improved the BER performance by 


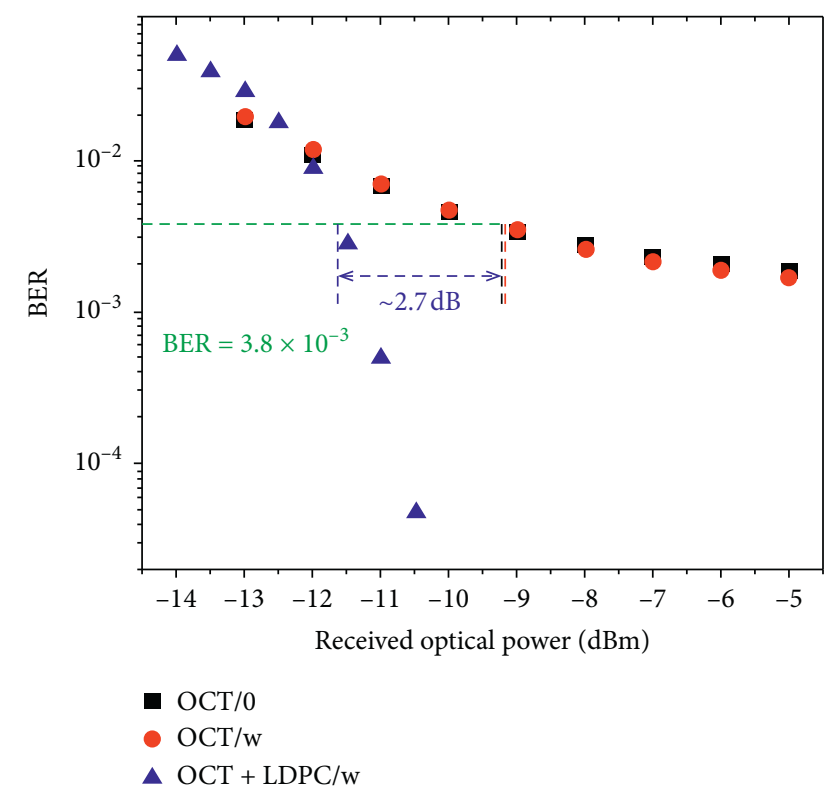

FIGURE 6: BER performance based on OCT-precoding combined with the LDPC-coding scheme.

about $2.7 \mathrm{~dB}$ but also averaged the SNR of the subcarriers, reducing the difference of each subcarrier in practical applications. However, it was not complete without shortcomings. The introduction of forward error correction technology caused signal redundancy and increased overhead. The LDPC-encoding rate we used was $87.5 \%$, which fully complied with the $20 \%$ overhead used for forward error correction.

\section{Conclusion}

The proposed scheme based on OCT-precoding combined with the LDPC-coding scheme could not only average the SNR of each subcarrier and reduce the difference of each subchannel in practical application but also improve the transmission performance of the system. The simulation results showed that the receiver sensitivity of the proposed scheme for 128 QAM MB-OFDM UWB signal was improved by $2.7 \mathrm{~dB}$ than that of the conventional scheme when the BER was $3.8 \times 10^{-3}$ after $70 \mathrm{~km}$ SSMF transmission. The LDPC-coding rate was $87.5 \%$, the net bit rate was $1.95 \mathrm{Gbps}$, and the spectral efficiency (SE) was $4.71 \mathrm{bit} / \mathrm{s} / \mathrm{Hz}$.

\section{Data Availability}

All the data used to support the findings of this study are available from the corresponding author upon request.

\section{Conflicts of Interest}

The authors declare that they have no conflicts of interest.

\section{Acknowledgments}

The authors really appreciate the support by the National Natural Science Foundation of China (61861019), the
Natural Science Foundation of Hunan Province of China (2019JJ50483), the project of Hunan Provincial Department of Education (18B316, 18C0588), and the Scientific Research Foundation of Hunan Province Higher Education Key Laboratory of Modeling and Monitoring on the Near-Earth Electromagnetic Environments (N201901).

\section{References}

[1] G. R. Aiello and G. D. Rogerson, "Ultra-wideband wireless systems," IEEE Microwave Magazine, vol. 4, no. 2, pp. 36-47, 2003.

[2] K. Gyouhwan, A. Rajeswaren, and R. Negi, "UWB versus 802.11-a network perspective," in Proceedings of the International Conference on Broadband Communications, Networks and Systems, IEEE, San Jose, CA, USA, October 2006.

[3] H.-H. Liu, W.-H. Lo, C.-C. Tseng, and H.-Y. Shin, "A WiFibased weighted screening method for indoor positioning systems," Wireless Personal Communications, vol. 79, no. 1, pp. 611-627, 2014.

[4] J. Perez and R. Llorente, "Hybrid UWB and WiMAX radioover-multi-mode fibre for in-building optical networks," Journal of Optics, vol. 16, no. 1, 2014.

[5] S. Pan and J. Yao, "Simultaneous provision of UWB and wired services in a WDM-PON network using a centralized light source," IEEE Photonics Journal, vol. 2, no. 5, pp. 712-718, 2010.

[6] T.-T. Pham, X. Yu, L. Dittmann, and I. T. Monroy, "Integration of optically generated impulse radio UWB signals into baseband WDM-PON," IEEE Photonics Technology Letters, vol. 23, no. 8, pp. 474-476, 2011.

[7] Y. Hong and L.-K. Chen, "Toward user mobility for OFDMbased visible light communications," Optics Letters, vol. 41, no. 16, pp. 3763-3766, 2016.

[8] Y. Hong, X. Guan, L. K. Chen et al., "Experimental demonstration of an OCT-based precoding scheme for visible light communications," in Optical Fiber Communication Conference/National Fiber Optic Engineers Conference, IEEE, Anaheim, CA, USA, March 2016.

[9] Y. Hong and L. K. Chen, "OCT precoding for OFDM-based indoor visible light communications," Optoelectronics and Communications Conference, pp. 3-5, 2016.

[10] S.-a. Liu, J. He, Q. Chen et al., "Experimental research of adaptive OFDM and OCT precoding with a high SE for VLLC system," Optical Fiber Technology, vol. 37, pp. 21-25, 2017.

[11] Y. Wei, J. He, R. Deng, J. Shi, S. Chen, and L. Chen, "An approach enabling adaptive FEC for OFDM in fiber-VLLC system," Optics Communications, vol. 405, pp. 329-333, 2017.

[12] J. He, C. Xiang, F. Long, K. Wu, and L. Chen, "Pre-compensation combined with TS-aided and ISFA-enhanced scheme for UWB system," Optics Communications, vol. 396, pp. 150-155, 2017.

[13] S. Bellini, M. Ferrari, A. Tomasoni, C. Costantini, L. Razzetti, and G. Gavioli, "LDPC design for block differential modulation in optical communications," Journal of Lightwave Technology, vol. 33, no. 1, pp. 78-88, 2015.

[14] IEEE 802.15.3 Working Group: Part 15.3: Wireless Medium Access Control (MAC) and Physical Layer (PHY) Specifications for High Data Rate Wireless Personal Area Networks (WPANs). http://www.iee802.org/15/pub/TG3.html,200910-12. 